\title{
Interaction Mechanism of Some Alkyl Iodides with Femtosecond Laser Pulses
}

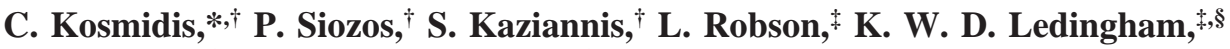 \\ P. McKenna, and D. A. Jaroszynski ${ }^{\ddagger}$ \\ Department of Physics, University of Ioannina, 45110 Ioannina, Greece, Department of Physics and \\ Applied Physics, University of Strathclyde, Glasgow G4 ONG, Scotland, U.K., and AWE Ltd., \\ Aldermaston, Reading RG7 4PR, U.K.
}

Received: October 4, 2004; In Final Form: November 30, 2004

\begin{abstract}
The interaction of 1-iodopropane, 2-iodopropane, 1-iodobutane, 2-iodobutane, and 1-iodopentane with $(5 \times$ $10^{13-} 5 \times 10^{15} \mathrm{~W} / \mathrm{cm}^{2}$ ) femtosecond laser fields is studied by means of a time-of-flight mass spectrometer. It is found that multiphoton ionization (MPI) and field ionization (FI) processes are involved in the molecular ionization. The contribution of these processes can be distinguished using the peak profile of the ions in the mass spectra. Thus, from the mass spectra of 2-iodoropane and 2-iodobutane, it is concluded that MPI processes are taking place even for Keldysh parameter values $\gamma \sim 0.3$. The field ionization process depends on the characteristics of the molecular binding potential well and leads to an asymmetric charge distribution of the transient multiply charged parent ions. In the case of 1-iodobutane, the MPI processes lead to a stable doubly charged parent ion production with a laser intensity threshold higher than that found for $\mathrm{I}^{2+}$ ions. In addition, the isomers studied exhibit distinct differences in their mass spectra and their origin is discussed in detail.
\end{abstract}

\section{Introduction}

Control of the dissociation process, molecular alignment, and high harmonic generation from molecular gases, induced by short and intense laser pulses, are some of the interesting phenomena that require a thorough understanding of the interaction mechanism between the molecular system and the laser beam. Although the interaction of strong laser beams $\left(10^{12-} 10^{17} \mathrm{~W} / \mathrm{cm}^{2}\right)$ with polyatomic molecules has a history of more than 10 years, many fundamental questions remain unanswered. It seems that the ionization/dissociation processes are better understood in the limits of the intensity range mentioned above. Thus, for the lower intensity limit $\left(\sim 10^{12}\right.$ $\mathrm{W} / \mathrm{cm}^{2}$ ) the ionization/dissociation processes can be understood by a multiphoton ionization mechanism (MPI), while for intensities above $10^{15} \mathrm{~W} / \mathrm{cm}^{2}$ molecular ionization is attributed to so-called "field ionization" i.e., ionization induced by the electric field of the laser beam (tunneling, barrier suppression ionization, electron recollision, etc).

On the other hand, the ionization/dissociation mechanism involved in experiments with laser intensities in the intermediate range $\left(10^{13-} 10^{15} \mathrm{~W} / \mathrm{cm}^{2}\right)$ is less clear, although it has been the subject of many recent publications. For atoms the criterion which defines the MPI and the field ionization (FI) regimes is the Keldysh parameter $\gamma\left[\gamma=\left(\mathrm{IP} / 2 U_{\mathrm{p}}\right)^{1 / 2}\right.$, where IP is the ionization potential and $U_{\mathrm{p}}$ is the ponderomotive potential of the free electron]. When $\gamma>1$, the ionization is attributed to MPI processes, while for $\gamma<1$ it is attributed to FI. In the case of polyatomic molecules the situation is much more complicated, and as Levis and co-workers have shown, the ionization in benzene can be attributed to FI even for values $\gamma$ $>3 .^{1}$

* Corresponding author. Fax: +30-26510-98695. E-mail: kkosmid@ cc.uoi.gr.

$\dagger$ University of Ioannina

$\doteqdot$ University of Strathclyde.

$\S$ AWE Ltd.
In addition, as far as molecular dissociation is concerned in this laser intensity region, Fuss et al. ${ }^{2}$ and Nakashima et al. ${ }^{3}$ have proposed that it is influenced by ionic state resonances which affect the abundance of parent ion in the mass spectra. The energy levels of the ionic states are determined from the photoelectron spectra of these molecules, and it is assumed that the laser field shifts them in parallel. Our collaboration group has shown that this criterion cannot be used universally, at least without some further considerations. In a series of molecules, we have shown that the parent ion production was not affected by resonances with ionic states. ${ }^{4}$

The exploration of the role of resonances with molecular states in ionization/dissociation processes is important. At first sight, their participation could be conceivable via "ladder climbing" and "ladder switching" processes, i.e., a multiphoton mechanism like that known from experiments with nanosecond and picosecond laser pulses..$^{5}$ On the other hand, Lezius et al. have suggested that in this intensity range "the discrete level structure that governs energy absorption in weak laser fields becomes irrelevant". 6 Undoubtedly, the picture of discrete energy levels is inadequate, since in polyatomic molecules the density of states is high and due to the Stark broadening the creation of a quasicontinuum of states is expected. Obviously, this does not mean that the interaction of strong laser beams with molecules is independent of the molecular electronic structure, and the opposite has been experimentally demonstrated in a series of publications. ${ }^{1,7-9}$ Assuming an efficient coupling of the ground state with the quasicontinuum of excited states through a "doorway state", the energy absorption is facilitated. Thus, the pumping up ("ladder climbing") way becomes very probable. This could account for the efficient ionization and intact parent ion production as reported in many femtosecond mass spectrometry works. ${ }^{10-12}$ Nevertheless, at the same time it is probable that the adiabatic potential surfaces can cross each other and nonadiabatic transitions between them can take place. The latter can result in the opening of various dissociation channels ("ladder switching"). 
To describe the interaction of strong laser fields with polyatomic molecules, some models have been proposed. We mention two of them, which have been published recently: an improved nonadiabatic multielectron model (NME) proposed by Markevitch et al. ${ }^{13}$ and that based on calculations using "field-following" time-dependent adiabatic states suggested by Kono et al. ${ }^{14}$ The latter has been applied in relevant lighter molecules compared to those studied by Markevitch et al. It seems that both models can successfully explain some of the observations reported by femtosecond experiments.

In the present work the interaction of a strong laser field (5 $\times 10^{13-5} \times 10^{15} \mathrm{~W} / \mathrm{cm}^{2}$ ) with iodo compounds is studied. In Scheme 1 the molecular structure of these species is presented, and it is clear that the size of alkyl chain varies. There are some reports claiming that the molecular coupling with the laser field increases with the molecular length. 6,15 On the other hand, other publications have demonstrated that there is no such straightforward dependence. ${ }^{2}$ To explore this, the molecules shown in Scheme 1 have been chosen. Moreover, we investigate the differences-if any-in the coupling of the laser field with isomeric molecular structures.

SCHEME 1: Diagram of (a) 1-Iodopropane $\left(1-\mathrm{C}_{3} \mathrm{H}_{7} \mathrm{I}\right)$, (b) 2-Iodopropane $\left(2-\mathrm{C}_{3} \mathrm{H}_{7} \mathrm{I}\right)$, (c) 1-Iodobutane $\left(1-\mathrm{C}_{4} \mathrm{H}_{9} \mathrm{I}\right)$, (d) 2-Iodobutane $\left(2-\mathrm{C}_{4} \mathrm{H}_{9} \mathrm{I}\right)$, and (e) 1-Iodopentane (1$\mathrm{C}_{5} \mathrm{H}_{11} \mathrm{I}$ )
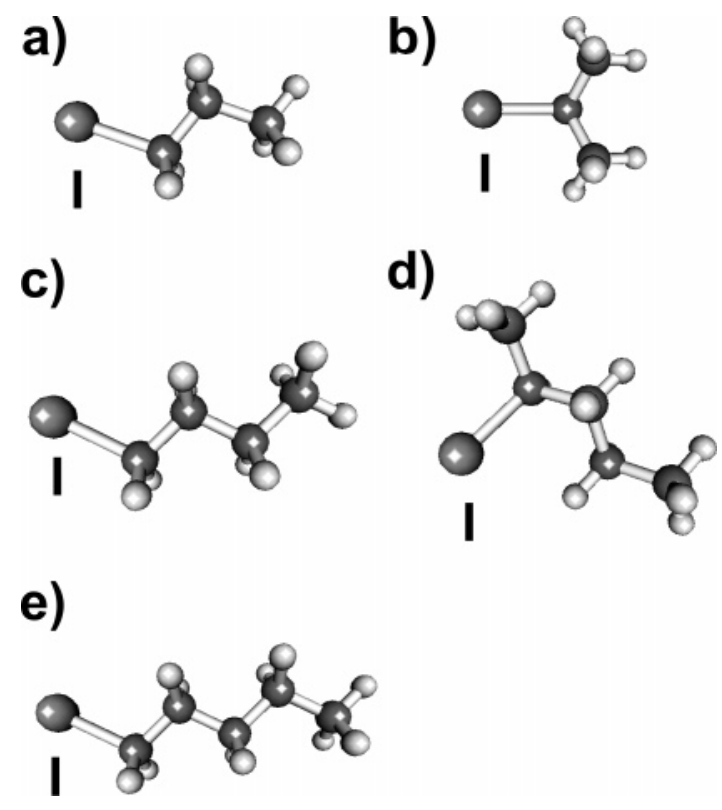

The motivation for the present work is to gain more insight about the interaction of molecules with lasers at high intensities.

\section{Experimental Details}

The reflectron time-of-flight (TOF) mass spectrometer used in the present study has been described in detail previously. ${ }^{16}$ Briefly, the system comprises a source chamber and flight tube, pumped using rotary-backed turbomolecular pumps to a base pressure of $1.3 \times 10^{-6} \mathrm{~Pa}$. The interaction chamber is spherical, $30 \mathrm{~cm}$ in diameter, and several ports fitted with fused quartz windows facilitate laser irradiation and direct sample viewing. Liquid samples for analysis are contained in glass vials coupled to a bleeder valve, and the vapors are admitted effusively to the source chamber via a capillary tube to a sample pressure lower than $4 \times 10^{-5} \mathrm{~Pa}\left(=3 \times 10^{-7}\right.$ Torr $)$. The microchannel plate (MCP) detector is contained in the $1.5 \mathrm{~m}$ flight tube. An ion optic arrangement based on Wiley-McLaren principles has been designed especially for this instrument to facilitate ion extraction and acceleration in electric fields. Potentials of +2.8 and $+2.3 \mathrm{kV}$ with respect to the field free flight tube are applied to the sample stage and ion optic, respectively. The ions are passed through the ion optic from a slit with $1 \mathrm{~mm}$ diameter and are accelerated into the reflectron electrostatic mirror before being detected by the MCP (Galileo). A digital oscilloscope (Tektronix TDS7104) is used for single-shot and averaged data collection and is connected to a PC.

Femtosecond ionization was performed using the Strathclyde Terahertz to Optical Pulse Source (TOPS) femtosecond laser system. ${ }^{17}$ To produce the seed pulse for the system, a Ti:sapphire Femtosource F220 laser was employed; this was pumped with a VERDI CW laser (532 nm, $5 \mathrm{~W})$. The repetition rate of the oscillator was $76 \mathrm{MHz}$ with an average power output of 500 $\mathrm{mW}$, generating pulses of $7 \mathrm{~nJ}$ with a pulse length of $20 \mathrm{fs}$. The low-energy, ultrashort pulses are then stretched to $200 \mathrm{ps}$ before being amplified in a $10 \mathrm{~Hz}, 10 \mathrm{~ns}$, Nd:YAG pumped regenerative Ti:sapphire amplifier. Two further multipass amplifiers generate the pulse of required energy: first a preamplifier, pumped by the same YAG as the regenerative amplifier; second a main amplifier pumped by two $10 \mathrm{~Hz}, 8 \mathrm{~W}$ SAGA YAGs. The amplified pulses are recompressed using a grating. This setup delivered linear polarized pulses of $250 \mathrm{~mJ}$, and temporal pulse widths (fwhm) of $60 \mathrm{fs}$ (as measured by an autocorrelator) at $800 \mathrm{~nm}$. A beam splitter provided the required energy, and variable attenuation of the beam $(0.1-9.0 \mathrm{~mJ})$ was achieved using absorptive neutral density filters.

The laser light was focused with a $25 \mathrm{~cm}$ focal length lens, and the polarization was rotated by using a half-wavelength plate at $800 \mathrm{~nm}$. In the present experiments the polarization angle is defined as the angle between the axis of the TOF and the polarization vector of the laser light. The intensities achieved at the focus were also checked through comparison with the intensities needed to produce multiple charged argon ions. ${ }^{18}$

\section{Results and Discussion}

From the absorption spectra ${ }^{19}$ it is known that the first absorption band of alkyl iodides lies in the region around 38500 $\mathrm{cm}^{-1}$. This corresponds to a valence transition, which is relatively weak and is assigned as an A-band. It has been reported by many dissociation studies that excitation to this state is followed by cleavage of the $\mathrm{C}-\mathrm{I}$ bond, ${ }^{20,21}$ and its lifetime for dissociation is about $125 \mathrm{fs}^{22}$

In the present experiments, the A-band can be reached after the absorption of three photons $(800 \mathrm{~nm})$, while for the ionization the absorption of at least three more photons is needed.

In Figure 1 the mass spectra recorded at laser intensities of $2.5 \times 10^{15} \mathrm{~W} / \mathrm{cm}^{2}$ are presented. There are some common characteristics among them: the parent ion peak (although with different relative intensity) and multiply charged atomic ions $\left(\mathrm{I}^{n+}, n \leq 7\right.$ and $\left.\mathrm{C}^{m+}, m \leq 4\right)$ are present in all mass spectra.

By varying the laser intensity in the range of $\sim 5 \times 10^{13-} 5$ $\times 10^{15} \mathrm{~W} / \mathrm{cm}^{2}$, the mass spectra of these molecules have been recorded. Thus, the laser intensity threshold for the appearance $\left(I_{\text {thr }}\right)$ of the multiply charged atomic ions can be determined, and these values are expected to be an upper intensity limit because their determination depends also on the acceptance efficiency of the ion optics used. These values are presented in Table 1 together with the intensity thresholds predicted by the barrier suppression ionization (BSI) model. For laser intensities above $\sim 8 \times 10^{13} \mathrm{~W} / \mathrm{cm}^{2}$ the Keldysh parameter is $\gamma<1$; 


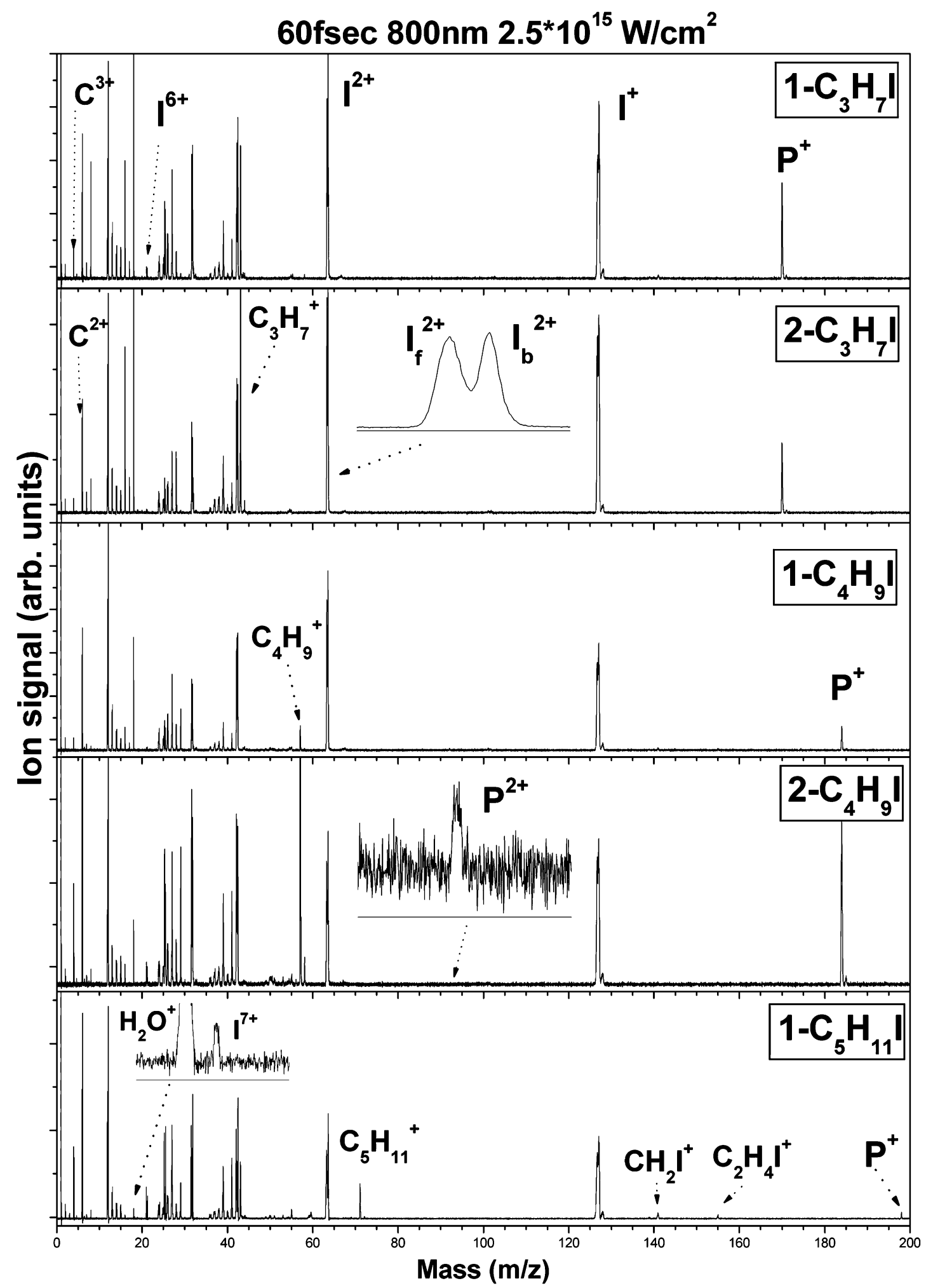

Figure 1. Mass spectra of alkyl iodides recorded at $800 \mathrm{~nm}$ using $60 \mathrm{fs}$ laser pulses $\left(I=2.5 \times 10^{15} \mathrm{~W} / \mathrm{cm}^{2}\right)$. The insert in the mass spectra of $2-\mathrm{C}_{3} \mathrm{H}_{7} \mathrm{I}$ shows the complex peak profile of $\mathrm{I}^{2+}$ ions (the $\mathrm{I}_{\mathrm{f}}^{2+}$ and $\mathrm{I}_{\mathrm{b}}{ }^{2+}$ in the insert denote the forward and backward components of the $\mathrm{I}^{2+}$ ion peak).

therefore, FI processes are expected to take place. The BSI is a simple model, and it has been applied successfully for atomic species $^{23}$ and for ionization of atomic fragments generated from molecular dissociation in picosecond experiments. ${ }^{24}$ The predicted intensity thresholds with this model can be calculated using the equation

$$
I\left(\mathrm{~W} / \mathrm{cm}^{2}\right)=\left(4 \times 10^{9}\right) E_{\mathrm{I}}^{4} / z^{2}
$$

where $E_{\mathrm{I}}$ is the ionization energy $(\mathrm{eV})$ of the atom (ion) and $z$ is the charge of the ionic state.

From Table 1, it is obvious that the intensity thresholds determined by experiment are much lower that those predicted by the BSI model. This implies that simple quasistatic models are inadequate and that the production of highly charged atomic ions can be achieved at lower laser intensities by irradiating molecular species. This could be attributed to the more effective 
TABLE 1: Intensity Threshold $\left(I_{\text {th }}\right)$ Values Predicted by BSI Model and Those Determined by the Present Work

\begin{tabular}{lllllllllll}
\hline & \multicolumn{1}{c}{$\mathrm{I}^{2+}$} & \multicolumn{1}{c}{$\mathrm{I}^{3+}$} & \multicolumn{1}{c}{$\mathrm{I}^{4+}$} & \multicolumn{1}{c}{$\mathrm{I}^{5+}$} & \multicolumn{1}{c}{$\mathrm{I}^{6+}$} & $\mathrm{I}^{7+}$ & \multicolumn{1}{c}{$\mathrm{C}^{+}$} & $\mathrm{C}^{2+}$ & $\mathrm{C}^{3+}$ & $\mathrm{C}^{4+}$ \\
\hline BSI model $\left(10^{14} \mathrm{~W} / \mathrm{cm}^{2+}\right)$ & 2.1 & 4.6 & 9.4 & 15 & 43 & 59 & 0.6 & 3.5 & 23 & 43 \\
1-iodopropane $\left(10^{14} \mathrm{~W} / \mathrm{cm}^{2}\right)$ & $0.5 \pm 0.1$ & $0.8 \pm 0.3$ & $1.5 \pm 0.3$ & $4.0 \pm 0.6$ & $20 \pm 4$ & & $0.5 \pm 0.1$ & $1.0 \pm 0.3$ & $10 \pm 1$ \\
2-iodopropane $\left(10^{14} \mathrm{~W} / \mathrm{cm}^{2}\right)$ & $0.5 \pm 0.1$ & $0.8 \pm 0.3$ & $1.2 \pm 0.6$ & $4.5 \pm 0.8$ & $8 \pm 3$ & & $0.5 \pm 0.1$ & $0.8 \pm 0.3$ & $4.5 \pm 0.8$ & $0.5 \pm 0.1$ \\
1-iodobutane $\left(10^{14} \mathrm{~W} / \mathrm{cm}^{2}\right)$ & $0.5 \pm 0.1$ & $0.5 \pm 0.1$ & $0.5 \pm 0.1$ & $2.6 \pm 0.2$ & $17 \pm 4$ & & $0.5 \pm 0.1$ & $0.5 \pm 0.1$ & $6.0 \pm 1.5$ & \\
2-iodobutane $\left(10^{14} \mathrm{~W} / \mathrm{cm}^{2}\right)$ & $<0.3$ & $0.4 \pm 0.1$ & $0.5 \pm 0.1$ & $0.9 \pm 0.3$ & $1.7 \pm 0.5$ & $15 \pm 2$ & $<0.3$ & $0.4 \pm 0.1$ & $0.9 \pm 0.3$ & $9.6 \pm 1.0$ \\
1-iodopentane $\left(10^{14} \mathrm{~W} / \mathrm{cm}^{2}\right)$ & $<0.4$ & $<0.4$ & $0.6 \pm 0.1$ & $0.9 \pm 0.3$ & $1.8 \pm 0.6$ & $8 \pm 2$ & $<0.4$ & $0.5 \pm 0.1$ & $0.9 \pm 0.3$ & $1.8 \pm 0.6$
\end{tabular}

TABLE 2: Estimated Kinetic Energy Values $(\mathrm{eV})$ of Ejected Fragment Ions at $I=6 \times 10^{15} \mathrm{~W} / \mathrm{cm}^{2}$

\begin{tabular}{|c|c|c|c|c|c|c|c|c|c|c|c|}
\hline & $\mathrm{I}^{+}$ & $\mathrm{I}^{2+}$ & $\mathrm{I}^{3+}$ & $\mathrm{I}^{4+}$ & $\mathrm{I}^{5+}$ & $\mathrm{I}^{6+}$ & $\mathrm{C}^{+}$ & $\mathrm{C}^{2+}$ & $\mathrm{C}^{3+}$ & $\mathrm{C}^{4+}$ & $\mathrm{H}^{+}$ \\
\hline 1-iodopropane & $0.9 \pm 0.1$ & $3.5 \pm 0.4$ & $9 \pm 0.9$ & $18 \pm 2$ & $30 \pm 3$ & $38 \pm 4$ & $8.0 \pm 1.0$ & $30 \pm 4$ & $79 \pm 9$ & & $16 \pm 5$ \\
\hline 2-iodopropane & $0.6 \pm 0.1$ & $2.1 \pm 0.3$ & $4.1 \pm 0.6$ & $7.3 \pm 1.1$ & $13 \pm 2$ & $19 \pm 3$ & $4.0 \pm 0.7$ & $17 \pm 3$ & $47 \pm 7$ & & $8 \pm 3$ \\
\hline 1-iodobutane & $0.8 \pm 0.1$ & $3.5 \pm 0.4$ & $8 \pm 0.9$ & $16 \pm 1.6$ & $25 \pm 3$ & $35 \pm 4$ & $8.0 \pm 0.9$ & $25 \pm 3$ & $67 \pm 8$ & & $16 \pm 5$ \\
\hline 2-iodobutane & $0.8 \pm 0.1$ & $5.2 \pm 0.5$ & $17 \pm 1.5$ & $28 \pm 2$ & $44 \pm 3$ & $55 \pm 5$ & $11.0 \pm 1.1$ & $49 \pm 5$ & $96 \pm 10$ & $126 \pm 15$ & $28 \pm 6$ \\
\hline 1-iodopentane & $1.5 \pm 0.1$ & $8.0 \pm 0.6$ & $24 \pm 2$ & $52 \pm 3$ & $79 \pm 5$ & $99 \pm 6$ & $19 \pm 2$ & $80 \pm 6$ & $169 \pm 13$ & $240 \pm 20$ & $37 \pm 7$ \\
\hline
\end{tabular}

coupling of a molecule with the laser field. If this argument is valid, then it is expected that the intensity thresholds for the multiply charged ions should decrease as the size of the alkyl chain increases. Indeed, from Table 1, the values determined for 1-iodopentane (the longest molecule studied in this work) are lower than those determined for 1-iodobutane and 1-iodopropane. At the same time the thresholds for 2-iodopropane and 2-iodobutane are found to be much lower that those of their isomers. The influence on $I_{\text {thr }}$ has the same significance as that of the alkyl chain length. Obviously, the structure of the isomer is equally as important as the molecular length.

All the atomic fragment ions exhibit a complex structure. Most of them have the characteristic "double peak" profile that is attributed to ions ejected initially away and toward the detector. The "double peak" profile of the ion peaks is considered as evidence of Coulomb explosion process that is taking place-due to strong repulsive forces-within a multiply charged molecular ion. The temporal separation between the two (backward and forward) components of the peak is frequently used for the estimation of the kinetic energies $\left(E_{\mathrm{kin}}\right)$ of the fragment ions in accordance with the following relation:

$$
E_{\text {kin }}(\mathrm{eV})=9.65 \times 10^{-7} \frac{\Delta t^{2} z^{2} F^{2}}{8 m}
$$

where $\Delta t(\mathrm{~ns})$ is the time difference between the forward and the backward components, $F(\mathrm{~V} / \mathrm{cm})$ is the electric field applied in the acceleration region of the TOF system, $z$ is the charge, and $m$ (amu) is the mass of the fragment ion.

The estimated $E_{\mathrm{kin}}$ values for the atomic fragment ions are presented in Table 2 . In an early work by Hatherly et al. ${ }^{25}$ it has been shown that the kinetic energy values for the atomic fragments, generated by Coulomb explosion within alkanes with different chain lengths, increase with the molecular size. The values of Table 2 support this trend.

As far as the peak profiles of the molecular fragment ions are concerned, some surprising results have been observed. Some of them exhibit a single peak profile. For instance, in the case of 1-iodopropane, the ions with $\mathrm{m} / \mathrm{z}=43\left(\mathrm{C}_{3} \mathrm{H}_{7}{ }^{+}\right), 41$ $\left(\mathrm{C}_{3} \mathrm{H}_{5}{ }^{+}\right), 39\left(\mathrm{C}_{3} \mathrm{H}_{3}{ }^{+}\right), 27\left(\mathrm{C}_{2} \mathrm{H}_{3}{ }^{+}\right)$, and $15\left(\mathrm{CH}_{3}{ }^{+}\right)$do not have the characteristic "double peak" profile of the other molecular fragments. Moreover, these peaks remain unchanged in the spectra when recorded with parallel and perpendicular polarization (Figure 2). This implies that these ions are not produced through a Coulomb explosion process and their kinetic energies are very small. The heaviest of these fragments corresponds to
I loss from the parent $(\mathrm{P})$ ion and it could be generated-in the 1-iodopropane case-through the reactions

$$
\begin{gathered}
\mathrm{P}^{+} \rightarrow \mathrm{C}_{3} \mathrm{H}_{7}^{+}+\mathrm{I} \\
{\left[\mathrm{P}^{2+}\right] \rightarrow \mathrm{C}_{3} \mathrm{H}_{7}^{+}+\mathrm{I}^{+}}
\end{gathered}
$$

Coulomb explosion in accordance to reaction 2 is expected to result in fragments with high kinetic energies (and "double peak" profiles); thus, it should be excluded as the source of the recorded $\mathrm{C}_{3} \mathrm{H}_{7}{ }^{+}$ions. The same is valid for fragmentation of higher charged unstable parent ions. It is probable that the large energy released by Coulomb explosion results in complete destruction of the $\mathrm{C}_{3} \mathrm{H}_{7}{ }^{+}$so that the recoil splitting cannot be observed with this ion. Obviously, the fragmentation of the $\mathrm{P}^{2+}$ ions can lead to $\mathrm{C}_{3} \mathrm{H}_{7}{ }^{+}$with a "single peak profile" only in the case of "smooth" dissociation, i.e., with very small recoil energy. The latter implies that different states of the $\mathrm{P}^{2+}$ ion can be reached, which is conceivable if a MPI mechanism is involved whereas there is no room for intermediate states in FI models.

Recently in the case of 1-iodopropane, M. S. Kim et al., using nanosecond lasers and the so-called mass-analyzed threshold ionization (MATI) technique, have reported conformer-specific dissociation pathways. ${ }^{26,27}$ For this specific molecule it is known that there are two conformers with $\mathrm{C}-\mathrm{C}-\mathrm{C}-\mathrm{I}$ torsion angles of $180^{\circ}$ (anti) and $66.1^{\circ}$ (gauche). ${ }^{28} \mathrm{Kim}$ and co-workers have found that in the case of anti conformer the cleavage of the $\mathrm{C}-\mathrm{I}$ bond in the ion is followed by a rearrangement in the $\mathrm{C}_{3} \mathrm{H}_{7}{ }^{+}$ leading to protonated cyclopropane (cyclo- $\mathrm{C}_{3} \mathrm{H}_{7}{ }^{+}$) formation. Similar rearrangement was not observed for the gauche conformer, where the "linear" structure of $\mathrm{C}_{3} \mathrm{H}_{7}+$ is preserved and $2-\mathrm{C}_{3} \mathrm{H}_{7}{ }^{+}$is produced. It has also been shown that the formation of protonated cyclopropane ion takes place rapidly in an excited state of the parent ion and it is less stable compared to that of $2-\mathrm{C}_{3} \mathrm{H}_{7}{ }^{+}$. In the gas phase 1-iodopropane exists as a mixture of the two conformers with the ratio of gauche:anti equal to 2:1. However, in the present work the conformer selectivity, reported by MATI experiments, is missing due to the fact that the available excess energy is expected to be higher than the typical barrier between the two conformers; therefore, the structure of the recorded $\mathrm{C}_{3} \mathrm{H}_{7}+$ ions remains insecure.

Regardless of the structure of the generated $\mathrm{C}_{3} \mathrm{H}_{7}{ }^{+}$ion, which needs further investigation, its fragmentation is likely to take place only after the end of the laser pulse and results in lighter ("single peak") molecular fragment production. By increasing the laser intensity, the lighter "single peak" molecular fragments 


\section{1- $\mathrm{C}_{3} \mathrm{H}_{7} \mathrm{I}:-$ Parallel Polarization}

\section{Perpendicular Polarization}

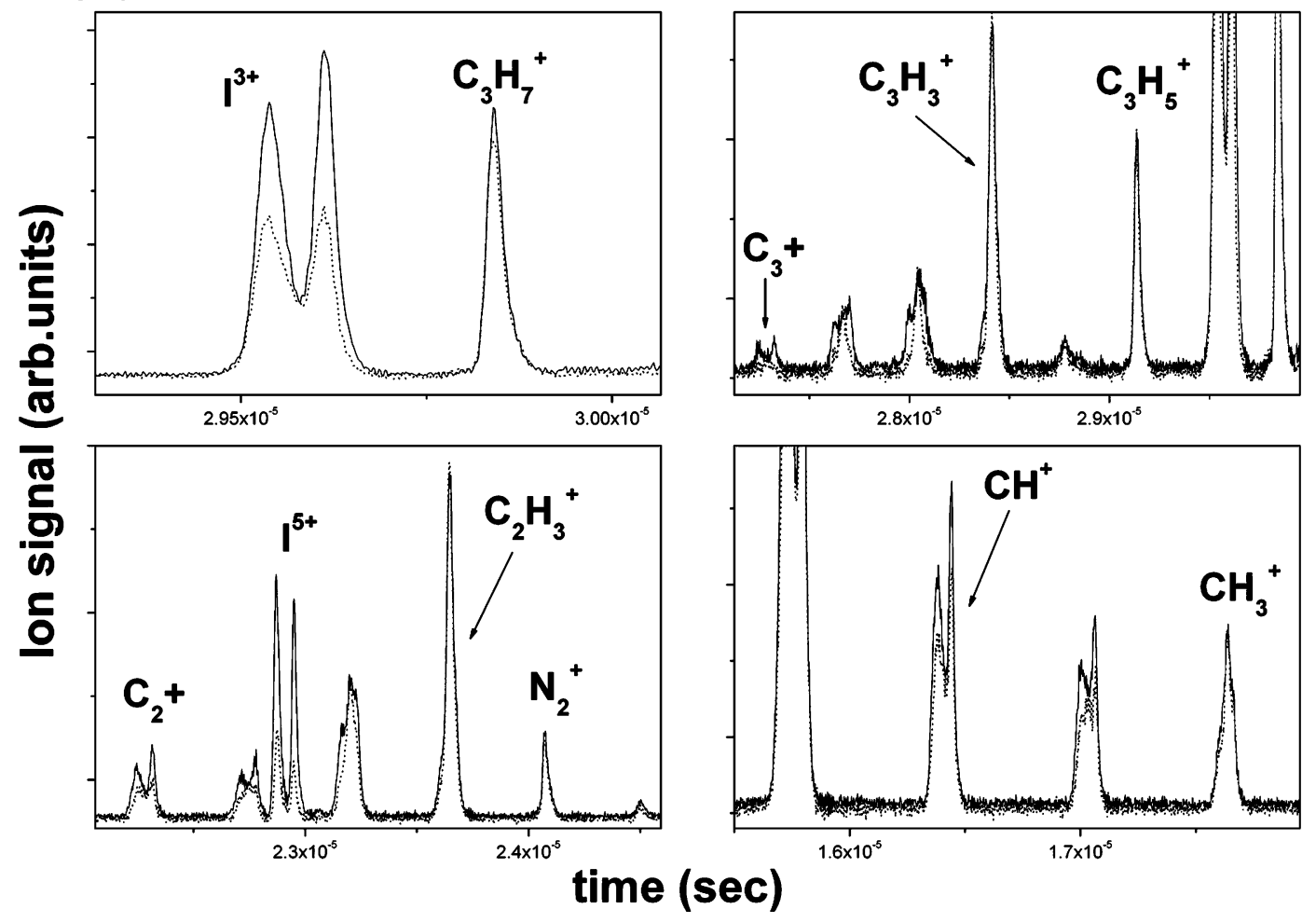

Figure 2. Peak profiles of $1-\mathrm{C}_{3} \mathrm{H}_{7} \mathrm{I}$ fragment ions recorded at $I=2.5 \times 10^{15} \mathrm{~W} / \mathrm{cm}^{2}$ with parallel and perpendicular laser polarization.

become stronger while the abundance of $\mathrm{C}_{3} \mathrm{H}_{7}+$ is still remarkable. It seems that as the internal energy of $\mathrm{C}_{3} \mathrm{H}_{7}+$ increases it breaks down to lighter fragments. Thus, the "single peak" profiles allow us the identification of the ionic fragmentation products of transient excited $\mathrm{C}_{3} \mathrm{H}_{7}{ }^{+}$(i.e., the $\mathrm{C}_{3} \mathrm{H}_{5}{ }^{+}, \mathrm{C}_{3} \mathrm{H}_{3}{ }^{+}$, $\mathrm{C}_{2} \mathrm{H}_{3}{ }^{+}$, and $\mathrm{CH}_{3}{ }^{+}$ions).

Obviously, this approach to explaining the experimental data relies on a multiphoton absorption in the ionic manifold, and it is in agreement with the appearance energies (AE) known from the literature ${ }^{29}$ for $\mathrm{C}_{3} \mathrm{H}_{7}{ }^{+}(\mathrm{AE}=9.84 \mathrm{eV})$ and $\mathrm{C}_{3} \mathrm{H}_{5}{ }^{+}(\mathrm{AE}=$ $11.23 \mathrm{eV}$ ) ions. The same observations about the I-loss ion are valid for the 1-iodobutane, too. In the case of 1-iodopentane, we have seen that the parent ion peak appears in the mass spectra at intensities higher than $10^{15} \mathrm{~W} / \mathrm{cm}^{2}$ and its amplitude increases with the laser intensity. Furthermore, we have noticed that the ratio of $[\mathrm{P}-\mathrm{I}]^{+}$ion peak over the total ion signal increases also (Figure 4).

In the mass spectra of 1-iodopentane, ion peaks, which have been assigned as $\mathrm{CH}_{2} \mathrm{I}^{+}$and $\mathrm{C}_{2} \mathrm{H}_{4} \mathrm{I}^{+}$, have been recorded. A small $\mathrm{CH}_{2} \mathrm{I}^{+}$ion peak exists also in the mass spectra of 1-iodopropane and 1-iodobutane, but there are no $\mathrm{C}_{x} \mathrm{H}_{y} \mathrm{I}^{+}$ion peaks in the mass spectra of their isomers. These ions are produced by the cleavage of a $\mathrm{C}-\mathrm{C}$ bond. The present experiments indicate that the rupture of a $\mathrm{C}-\mathrm{C}$ bond becomes more efficient as the length of the alkyl chain increases. Ross and Johnston ${ }^{20}$ have studied the photochemistry of iodoalkanes using nanosecond laser pulses at 248 and $193 \mathrm{~nm}$. They found that molecular dissociation by $\mathrm{C}-\mathrm{C}$ bond cleavage takes place only at $193 \mathrm{~nm}$. The fragmentation channels induced by strong picosecond and femtosecond laser fields at different wavelengths will be discussed in detail in a forthcoming paper.

Moreover, in 1-iodobutane a peak at $\mathrm{m} / \mathrm{z}=92$ assigned as double charged parent ion $\left(\mathrm{P}^{2+}\right)$ has been recorded. The intensity threshold for the appearance of this ion has been found to be
$I_{\text {thr }} \sim 8 \times 10^{13} \mathrm{~W} / \mathrm{cm}^{2}$. This intensity value lies in that socalled borderline region between MPI and FI processes. The ionization potential (IP) for the $\mathrm{P}^{2+}$ of 1-iodobutane can be estimated by the approximate relation ${ }^{30} \mathrm{IP}^{2+}=2.8(\mathrm{IP}) \sim 26$ $\mathrm{eV}$. For this $\mathrm{IP}^{2+}$ value and laser intensity $I_{\mathrm{thr}} \sim 8 \times 10^{13} \mathrm{~W} / \mathrm{cm}^{2}$, the Keldysh parameter value is $\gamma \sim 3.2>1$. Thus, the production of $\mathrm{P}^{2+}$ via MPI processes cannot a priori be excluded, although as mentioned before the ionization of benzene at similar $\gamma$ values has been attributed to the FI mechanism. ${ }^{1}$ Nevertheless, it should be noted that the observation of $\mathrm{P}^{2+}$ in the case of 1,3-cyclohexadiene ${ }^{2}$ and in thiazolidine ${ }^{8}$ at similar laser intensities has been attributed to MPI processes. For the case of 1-iodobutane, it is surprising that the $I_{\text {thr }}$ of $\mathrm{P}^{2+}$ is higher than those found for $\mathrm{I}^{+}, \mathrm{I}^{2+}$, and $\mathrm{C}^{+}$ions and similar to that of the $\mathrm{I}^{3+}$ ion. This is conceivable only if it is accepted that in laser intensities lower than that of the $I_{\text {thr }}$ of $\mathrm{P}^{2+}$ a mechanism which leads to unstable multiply charged parent ions $\left(\left[\mathrm{P}^{n+}\right], n>2\right)$ is in operation. Since, under the present experimental conditions (the dissociation lifetime ${ }^{22}$ is longer than the pulse duration), these atomic ions cannot be produced by a process that requires molecular fragmentation followed by MPI of the atomic fragments, the mechanism involved in their generation should be an FI one.

Furthermore, from Table 1, it is clear that the $I_{\text {thr }}$ for $\mathrm{I}^{6+}$ is lower than that of $\mathrm{C}^{4+}$ although the energy needed for $\mathrm{C}^{4+}$ formation is $64.5 \mathrm{eV}$, while for $\mathrm{I}^{6+}$ it is $78.8 \mathrm{eV} .^{31}$ This implies that the charge distribution on the molecular skeleton prior to the fragmentation is asymmetric; i.e., the charge state of I atoms is higher than that of each carbon atom. The asymmetric charge distribution is in agreement with those reported by Strickland et al. ${ }^{32}$ and Posthumus et al. ${ }^{33}$ for the case of $\mathrm{I}_{2}$. It should be mentioned that charge asymmetric fragmentation was observed when lasers with pulse duration shorter $(\leq 200 \mathrm{fs})$ than the dissociation lifetime were used. 


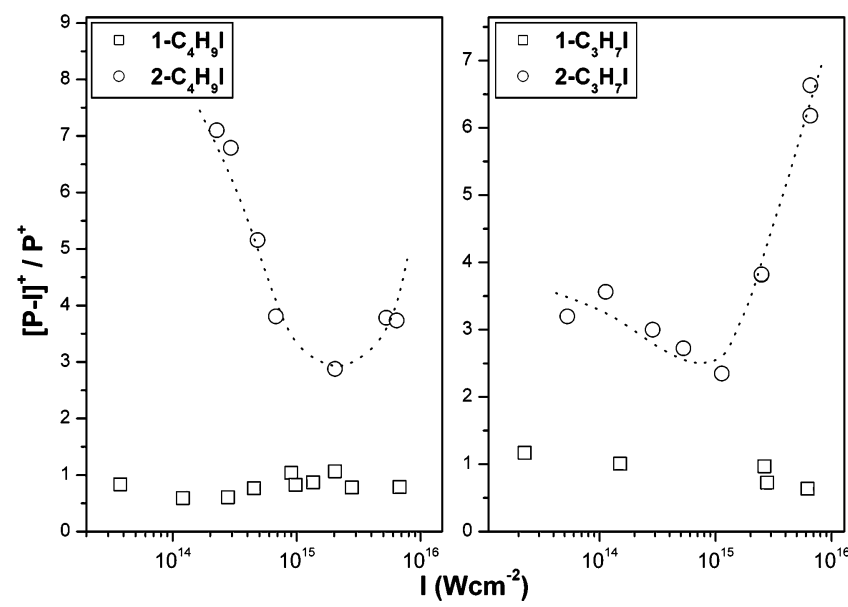

Figure 3. Intensity dependence of the ratio of $[\mathrm{P}-\mathrm{I}]^{+} / \mathrm{P}^{+}$ion signal.

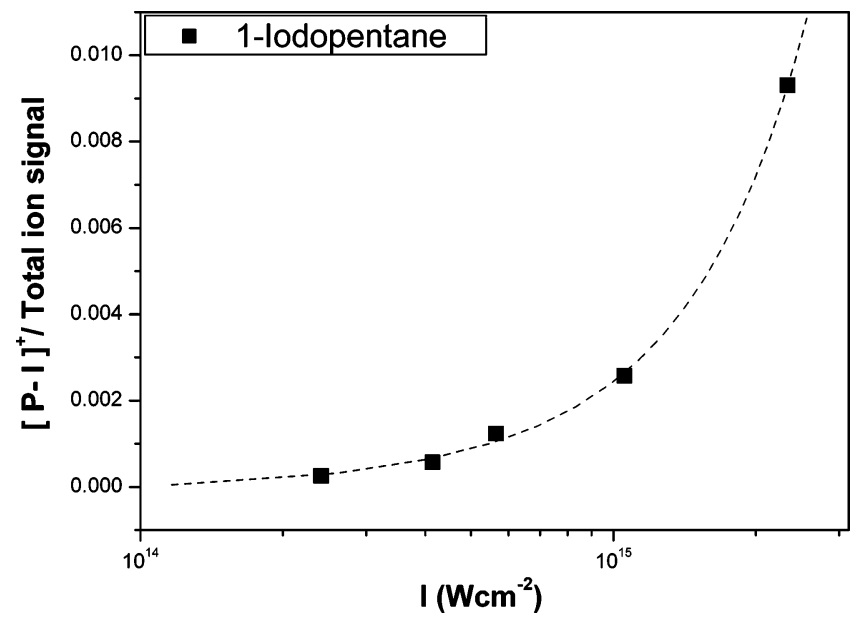

Figure 4. Intensity dependence of the ratio of $[\mathrm{P}-\mathrm{I}]^{+}$over total ion signal for 1-iodopentane.

Therefore, the interpretation of the findings of the present work cannot be explained by a single ionization mechanism. It seems that both MPI and FI processes are in operation in this intensity region. The peak profiles of the recorded ions can be used for the identification of the MPI process. The latter lead to $\mathrm{P}^{2+}$ ion production (stable in 1-iodobutane) by a "ladder climbing" process facilitated by the creation of a quasicontinuum due to the broadening of the ionic states by the strong laser field. The route to the $\mathrm{P}^{2+}$ formation is interrupted-"ladder switching"-in some ions and gives rise to I-loss ions ([P I]). Depending on the number of photons absorbed by the parent ion prior to the $\mathrm{C}-\mathrm{I}$ bond cleavage, the $[\mathrm{P}-\mathrm{I}]$ ions are generated in excited states and fragment. The fragmentation channels can be verified by checking the profile of some molecular fragments ("single peak" profiles).

At the same time FI is taking place, too. Unstable parent ions with asymmetric charge distribution are generated which subsequently fragment due to strong Coulombic repulsion.

The above description of the interaction processes of the molecules with the laser field is strengthened by taking into account the data observed for the two isomers (2-iodopropane and 2-iodobutane), too. The most striking difference in the mass spectra (Figure 1) between the isomers is the intensity of I-loss $([\mathrm{P}-\mathrm{I}])$ ion peak. This fragment ion is the base peak in the mass spectra of 2-iodopropane and 2-iodobutane. In Figure 3 the dependence of the ratio of the peaks $[\mathrm{P}-\mathrm{I}]^{+} / \mathrm{P}^{+}$on the laser intensity is presented. For 1-iodopropane and 1-iodobutane the ratio versus laser intensity remains almost constant, within experimental error. However, it is surprising that for 2-iodopropane and 2-iodobutane the ratio value decreases as the laser intensity increases in the range of $\sim 10^{14-} 10^{15} \mathrm{~W} / \mathrm{cm}^{2}$. This observation cannot be explained by assuming resonances in the ionic manifold since the photoelectron spectra of these molecules $^{34}$ reveal a similar structure for the isomers and the small energy differences between the ionic states are within the laser bandwidth.

However, the $[\mathrm{P}-\mathrm{I}]^{+} / \mathrm{P}^{+}$ratio dependence is conceivable by taking into account the three-photon excitation to the dissociative A-state of the neutral molecules. Molecules in this state can either be ionized by further three-photon absorption (direct vertical ionization) or relax and some of them ultimately fragment. During relaxation a part of the absorbed energy is transferred to vibrational degrees of freedom of the intact molecule, resulting in the production of excited "hot" species. The $\mathrm{C}-\mathrm{I}$ bond strength is lower in 2-iodoalkanes compared to their isomers and their dissociation gives rise to $[\mathrm{P}-\mathrm{I}]$ neutral fragments. Thus, in the A-state there are two competing processes. By increasing laser intensity, the direct ionization rate increases, which implies that the ratio $[\mathrm{P}-\mathrm{I}]^{+} / \mathrm{P}^{+}$decreases.

On the other hand, for even higher laser intensities other excitation processes are becoming probable. Hence, the absorption of more than three photons by the unrelaxed molecules can lead to electronically excited $\mathrm{P}^{+}$ions, which subsequently fragment (reaction 1). At the same time, the ionization of the relaxed "hot" molecules becomes also probable resulting thus in "hot" unstable $\mathrm{P}^{+}$ions generation, which are expected also to dissociate to $[\mathrm{P}-\mathrm{I}]^{+}$. It is of importance to note that the produced $[\mathrm{P}-\mathrm{I}]^{+}$ions from these processes are expected to have small recoil energy (i.e., "single peak profile") and that both of them imply that the ratio $[\mathrm{P}-\mathrm{I}]^{+} / \mathrm{P}^{+}$increases with laser intensity, in agreement with that experimentally observed for laser intensities $>10^{15} \mathrm{~W} / \mathrm{cm}^{2}$ (Figure 3).

Although it cannot be a priori excluded, the vertical excitation to ionic electronic states of the unrelaxed molecules is not expected to be the main reason that accounts for the observed difference between the isomers as far as the dependence of the $[\mathrm{P}-\mathrm{I}]^{+} / \mathrm{P}^{+}$ratio on laser intensity is concerned. This is because the difference in the direct excitation rate from the A-state of the isomers is expected to be small. Therefore, the reason for the different dependence of the $[\mathrm{P}-\mathrm{I}]^{+} / \mathrm{P}^{+}$ratio should be ascribed to the ionization of the relaxed intact molecules (i.e., to nonvertical excitations). It is known that 2-iodoalkanes stretch the $\mathrm{C}-\mathrm{I}$ bond faster than the 1-iodoalkanes, which means that the relaxation rate in the A-state is higher for the former molecules. This different rate of the isomers in the population leakage from the A-state to excited states-in the neutral manifold-out of the Franck-Condon region can account for the different dependence of the $[\mathrm{P}-\mathrm{I}]^{+} / \mathrm{P}^{+}$ratio on laser intensity. Obviously, these nonvertical transitions are conceivable only in the MPI regime.

The above analysis shows that multiphoton processes are taking place even at $10^{15} \mathrm{~W} / \mathrm{cm}^{2}$ (i.e., for $\gamma \sim 0.3$ ) -in parallel with FI-and MPI is the dominant mechanism as far as the production of some ions is concerned. It may be argued that the observed multiphoton processes are taking place in the spatial wings of the focused laser beam as we have proposed in some previous publications. ${ }^{36,37}$ However, in the present set of experiments, a narrow slit $(1 \mathrm{~mm})$ was inserted before the ion optics in order to eliminate the contribution from the wings; in any case this dependence was not observed for 1-iodopropane and 1-iodobutane mass spectra recorded by the same experimental setup. 


\section{Conclusions}

The main conclusion with respect to the ionization/dissociation mechanism involved in the present experiments is that for laser intensities in the range $10^{14-} 10^{15} \mathrm{~W} / \mathrm{cm}^{2}$ both MPI and FI processes contribute to molecular ionization.

The multiphoton processes ("ladder climbing") in this intensity range are facilitated by the creation of a quasicontinuum caused by Stark broadening of the molecular states. Dissociation channels in the neutral and ionic manifolds affect the "climbing" to single and/or double ionization. The dissociation in the neutral molecules due to $\mathrm{C}-\mathrm{I}$ bond cleavage was found to be a ratelimiting step for the ionization in the case of 2-iodopropane and 2-iodobutane.

The characteristic fingerprint of the MPI processes is the "single component" peaks in the mass spectra. This feature could be used for the identification of the dissociation channels of transient excited fragment ions produced during the interaction with the laser field. At the same time FI processes are also taking place. For the molecules studied in the present work, it is concluded that the produced multiply charged transient parent ions have an asymmetric charge distribution prior to their dissociation. The generated multiply charged atomic ions have a "double peak" profile, and their estimated kinetic energies imply that the molecular coupling with the laser field increases with the molecular length. Moreover, the molecular length is not the only parameter that affects the coupling with the field. From the comparison of the $I_{\text {thr }}$ values for the multiply charged atomic ions, it is found that these values are lower for 2-iodopropane and 2-iodobutane compared to their isomers.

As far as the comparison of the interaction with the laser field of the isomers studied in the present work is concerned, we can conclude that there are some distinct differences: the MPI processes lead to a higher abundance of the $[\mathrm{P}-\mathrm{I}]$ ion in the mass spectra of 2-iodopropane and 2-iodobutane compared to those of their isomers, while the dependence of this ion on laser intensity is totally different; the multiply charged atomic ions generated by FI processes are found to have higher kinetic energies and lower intensity thresholds in the cases of 2-iodopropane and 2-iodobutane. The latter is thought to be an indication that the coupling of these molecules with the laser field is more effective compared to that of their isomers.

In agreement with previous reports, ${ }^{6,35,36}$ the analysis of the experimental data confirms that the quasistatic models are inadequate for the interpretation of the molecular coupling with strong laser fields. The intensity thresholds for the generation of multiply charged atomic ions are found to be much lower than those predicted by the BSI model while part of the molecular ionization and dissociation is attributed to multiphoton processes even for laser intensities where the Keldysh parameter has values $\gamma \sim 0.3<1$.

Obviously, the above interpretation of the molecular interaction with the laser field does not form a complete and universal model and further theoretical and experimental work is needed for the creation of such a model.

Acknowledgment. We express our thanks to the staff and administration of the TOPS Lab of the Strathclyde University and the Central Laser Facility of the University of Ioannina for their facilities and their assistance. This research was funded by the program "Heraklitos" of the Operational Program for Education and Initial Vocational Training of the Hellenic Ministry of Education under the Third Community Support Framework and the European Social Fund.

\section{References and Notes}

(1) Levis, R. J.; Dewitt, M. J. J. Phys. Chem. A 1999, 103, 64936507.

(2) Fuss, W.; Schmid, W. E.; Trushin, S. A. J. Chem. Phys. 2000, 112, $8347-8362$

(3) Harada, H.; Shimizu, S.; Yatsuhashi, T.; Sakabe, S.; Izawa, Y.; Nakashima, N. Chem. Phys. Lett. 2001, 342, 563-570.

(4) Robson, L.; Ledingham, K. W. D.; Tasker, A. D.; McKenna, P.; McCanny, T.; Kosmidis, C.; Jaroszynski, D. A.; Jones, D. R.; Issac, R. C.; Jamieson, S. Chem. Phys. Lett. 2002, 360, 382-389.

(5) Boesl, U. J. Phys. Chem. 1991, 95, 2949-2962.

(6) Lezius, M.; Blanchet, V.; Rayner, D. M.; Villeneuve, D. M.; Stolow, A.; Ivanov, M. Y. Phys. Rev. Lett. 2001, 86, 51-54.

(7) Kosmidis, C.; Tzallas, P.; Ledingham, K. W. D.; McCanny, T.; Singhal, R. P.; Taday, P. F.; Langley, A. J. J. Phys. Chem. A 1999, 103, 6950-6955.

(8) Tzallas, P.; Kosmidis, C.; Philis, J. G.; Ledingham, K. W. D.; McCanny, T.; Singhal, R. P.; Hankin, S. M.; Taday, P. F.; Langley, A. J. Chem. Phys. Lett. 2001, 343, 91-98.

(9) Dewitt, M. J.; Levis, R. J. J. Chem. Phys. 1998, 108, 7739-7742.

(10) Ledingham, K. W. D.; Kilic, H. S.; Kosmidis, C.; Deas, R. M.; Marshall, A.; McCanny, T.; Singhal, R. P.; Langley, A. J.; Shaikh, W. Rapid Commun. Mass Spectrom. 1995, 9, 1522-1527.

(11) Kilic, H. S.; Ledingham, K. W. D.; Kosmidis, C.; McCanny, T.; Singhal, R. P.; Wang, S. L.; Smith, D. J.; Langley, A. J.; Shaikh, W. J. Phys. Chem. A 1997, 101, 817-823.

(12) Grun, C.; Heinicke, R.; Weickhardt, C.; Grotemeyer, J. Int. J. Mass Spectrom. 1999, 185-187, 307-318.

(13) Markevitch, A. N.; Romanov, D. A.; Smith, S. M.; Schlegel, H. B.; Ivanov, M. Y.; Levis, R. J. Phys. Rev. A 2004, 69, art. no. 013401.

(14) Kono, H.; Sato, Y.; Tanaka, N.; Kato, T.; Nakai, K.; Koseki, S.; Fujimura, Y. Chem. Phys. 2004, 304, 203-226.

(15) Dewitt, M. J.; Levis, R. J. J. Chem. Phys. 1999, 110, 11368-11375.

(16) Tasker, A. D.; Robson, L.; Ledingham, K. W. D.; McCanny, T.;

Hankin, S. M.; McKenna, P.; Kosmidis, C.; Jaroszynski, D. A.; Jones, D. R. J. Phys. Chem. A 2002, 106, 4005-4013.

(17) Jaroszynski, D. A.; Ersfeld, B.; Giraud, G.; Jamison, S.; Jones, D. R.; Issac, R. C.; Mcneil, B. M. W.; Phelps, A. D. R.; Robb, G. R. M.; Sandison, H.; Vieux, G.; Wiggins, S. M.; Wynne, K. Nucl. Instrum. Methods Phys. Res., Sect. A 2000, 445, 317-319.

(18) Auguste, T.; Monot, P.; Lompre, L. A.; Mainfray, G.; Manus, C. J. Phys. B: At. Mol. Opt. Phys. 1992, 25, 4181-4194.

(19) Robin, M. B. Higher Excited States of Polyatomic Molecules; Academic Press: New York and London, 1974; Vol. 1.

(20) Ross, P. L.; Johnston, M. V. J. Phys. Chem. 1995, 99, 4078-4085.

(21) Szaflarski, D. M.; El-Sayed, M. A. J. Phys. Chem. 1988, 92, 22342239

(22) Zhong, D. P.; Zewail, A. H. J. Phys. Chem. A 1998, 102, 40314058

(23) Augst, S.; Meyerhofer, D. D.; Strickland, D.; Chin, S. L. J. Opt. Soc. Am. B: Opt. Phys. 1991, 8, 858-867.

(24) Siozos, P.; Kaziannis, S.; Kosmidis, C. Int. J. Mass Spectrom. 2003, $225,249-259$.

(25) Hatherly, P. A.; Frasinski, L. J.; Codling, K.; Barr, J. R. M. Chem. Phys. Lett. 1988, 149, 477-481.

(26) Park, S. T.; Kim, S. K.; Kim, M. S. Nature 2002, 415, 306-308

(27) Park, S. T.; Kim, M. S. J. Chem. Phys. 2002, 117, 124-131.

(28) Hagen, K.; Stolevik, R.; Saebo, P. C. J. Mol. Struct. 1995, 346, $75-82$.

(29) NIST Chemistry WebBook; http://webbook.nist.gov.

(30) Tsai, B. P.; Eland, J. H. D. Int. J. Mass Spectrom. Ion Process. 1980, 36, 143-165.

(31) Ford, J. V.; Zhong, Q.; Poth, L.; Castleman, A. W. J. Chem. Phys. 1999, 110, 6257-6267.

(32) Strickland, D. T.; Beaudoin, Y.; Dietrich, P.; Corkum, P. B. Phys. Rev. Lett. 1992, 68, 2755.

(33) Posthumus, J. H.; Giles, A. J.; Thompson, M. R.; Colding, K. J. Phys. B: At. Mol. Opt. Phys. 1996, 29, 5811-5829.

(34) Kimura, K.; Katsumata, S.; Achiba, A.; Yamazaki, T.; Iwata, S. Handbook of HeI photoelectron spectra of fundamental molecules; Japan Scientific Society Press: Tokyo, 1981

(35) Trushin, S. A.; Fuss, W.; Schmid, W. E. J. Phys. B: At. Mol. Opt. Phys. 2004, 37, 3987-4011.

(36) Tzallas, P.; Kosmidis, C.; Ledingham, K. W. D.; Singhal, R. P.; McCanny, T.; Graham, P.; Hankin, S. M.; Taday, P. F.; Langley, A. J. J. Phys. Chem. A 2001, 105, 529-536.

(37) Ledingham, K. W. D.; Smith, D. J.; Singhal, R. P.; McCanny, T.; Graham, P.; Kilic, H. S.; Peng, W. X.; Langley, A. J.; Taday, P. F.; Kosmidis, C. J. Phys. Chem. A 1999, 103, 2952-2963.

(38) Markevitch, A. N.; Romanov, D. A.; Smith, S. M.; Levis, R. J. Phys. Rev. Lett. 2004, 92, art. no. 063001. 